\title{
The role of Sox6 in zebrafish muscle fiber type specification
}

\author{
Harriet E Jackson ${ }^{1,2}$, Yosuke Ono ${ }^{1}$, Xingang Wang ${ }^{1}$, Stone Elworthy ${ }^{2}$, Vincent T Cunliffe ${ }^{2}$ and Philip W Ingham ${ }^{1,3,4^{*}}$
}

\begin{abstract}
Background: The transcription factor Sox6 has been implicated in regulating muscle fiber type-specific gene expression in mammals. In zebrafish, loss of function of the transcription factor Prdm1a results in a slow to fast-twitch fiber type transformation presaged by ectopic expression of sox6 in slow-twitch progenitors. Morpholino-mediated Sox6 knockdown can suppress this transformation but causes ectopic expression of only one of three slow-twitch specific genes assayed. Here, we use gain and loss of function analysis to analyse further the role of Sox6 in zebrafish muscle fiber type specification.

Methods: The GAL4 binary misexpression system was used to express Sox6 ectopically in zebrafish embryos. Cis-regulatory elements were characterized using transgenic fish. Zinc finger nuclease mediated targeted mutagenesis was used to analyse the effects of loss of Sox6 function in embryonic, larval and adult zebrafish. Zebrafish transgenic for the GCaMP3 Calcium reporter were used to assay Ca2+ transients in wild-type and mutant muscle fibres.
\end{abstract}

Results: Ectopic Sox6 expression is sufficient to downregulate slow-twitch specific gene expression in zebrafish embryos. Cis-regulatory elements upstream of the slow myosin heavy chain 1 (smyhcl) and slow troponin c (tnnclb) genes contain putative Sox6 binding sites required for repression of the former but not the latter. Embryos homozygous for sox6 null alleles expressed tnncib throughout the fast-twitch muscle whereas other slow-specific muscle genes, including smyhc1, were expressed ectopically in only a subset of fast-twitch fibers. Ca2+ transients in sox6 mutant fast-twitch fibers were intermediate in their speed and amplitude between those of wild-type slow- and fast-twitch fibers. sox6 homozygotes survived to adulthood and exhibited continued misexpression of tnnc $1 b$ as well as smaller slow-twitch fibers. They also exhibited a striking curvature of the spine.

Conclusions: The Sox6 transcription factor is a key regulator of fast-twitch muscle fiber differentiation in the zebrafish, a role similar to that ascribed to its murine ortholog.

Keywords: zebrafish, muscle, fiber type, Sox6, troponin, myosin, spinal curvature

\section{Background}

Vertebrate skeletal muscle is composed of distinct fiber types that differ in their physiological and metabolic properties; Type I or slow-twitch fibers have a low contraction velocity but are rich in mitochondria and are therefore more efficient at using oxygen to generate ATP, resulting in a high endurance capability. Type II or fast-twitch fibers, by contrast, are more suited to generating short burst of strength or speed, but they fatigue more rapidly

\footnotetext{
* Correspondence: pingham@imcb.a-star.edu.sg

${ }^{1} A^{*}$ STAR Institute of Molecular and Cell Biology, Proteos, 61 Biopolis Drive, Singapore 138673, Republic of Singapore

${ }^{3}$ Lee Kong Chian School of Medicine, Nanyang Technological University, Proteos, 61 Biopolis Drive, Singapore 138673, Republic of Singapore Full list of author information is available at the end of the article
}

than slow-twitch fibers due to their high contraction velocity. Although largely genetically determined, the fiber type composition of muscles is also partially adaptive; endurance training by long distance runners, for instance, can increase their proportion of Type I fibers through conversion of Type IIa fibers. Most studies of the control of muscle fiber type in mammals have focused on their activity dependent diversity and plasticity [1]; by contrast, rather less is known about the allocation of myoblasts to distinct fates during embryonic development.

The zebrafish provides a highly tractable model to study vertebrate fiber type specification, as the embryonic myotome shows a discrete temporal and spatial separation of fiber type ontogeny that facilitates genetic analysis of its 
development [2]. Zebrafish myogenesis begins prior to somite formation with the activation of the myogenic regulatory factors (MRFs), myoD and myf5 [3-6] The cells closest to the notochord, the so-called adaxial cells [7], are the first myoblasts to be specified and begin to differentiate prior to somitogenesis in response to notochord-derived Hedgehog $(\mathrm{Hh})$ signals [4,8-13]. Most adaxial cells elongate and migrate radially outward to form a subcutaneous layer of mononucleated slow-twitch muscle fibers named superficial slow-twitch fibers (SSF) [7]. A specialized subpopulation of adaxial cells, the muscle pioneers (MPs) are characterized by their expression of the Engrailed transcription factors and retain their medial location to form the horizontal myoseptum that subdivides the myotome into dorsal (epaxial) and ventral (hypaxial) compartments $[7,14,15]$. The bulk of the myotome comprises the fast-twitch fibers, which begin their differentiation in the wake of the migrating slow-twitch fibers $[4,16]$. The fast muscle progenitors mature and fuse with each other to form a multinucleated array of syncytial fibers [13].

The Sry transcription family member Sox6 has been implicated in muscle fiber type specification in both mice and fish. Mice mutant for sox6 display an increase in slowspecific gene expression and a concomitant decrease in the expression of fast-twitch specific genes $[17,18]$, suggesting that Sox6 normally functions to promote the fast-twitch differentiation program and repress slow-specific gene expression in fetal muscle fibers. Consistent with this, ChIPseq analysis has revealed the direct interaction of Sox6 with the regulatory elements of slow-specific genes in mice $[19,20]$. In zebrafish embryos lacking activity of the Prdm1a transcription factor, adaxial cells differentiate into fast-twitch fibers, a transformation that is accompanied by the ectopic expression of sox6. Transient knockdown of Sox6 mediated by morpholino antisense oligonucleotides is sufficient to suppress this transformation, suggesting that similar to its role in mouse, Sox6 normally acts to repress slow-twitch gene expression in zebrafish [21]. Surprisingly, however, while tnnc1b is de-repressed in the fast fibers of Sox6 morphant embryos, no ectopic smyhc1 expression was observed. This could reflect an incomplete inactivation of Sox6 function achieved by morpholinos or indicate a different pathway of repression and/or activation of smyhc1. Here, we have used targeted overexpression and mutagenesis of the sox 6 gene to explore further its role in zebrafish muscle fiber type specification. Our findings confirm and extend the results of our previous transient knock-down studies and imply that Sox6 is not the sole mediator of slow-twitch gene repression.

\section{Methods}

\section{Ethics statement}

The research described in this paper uses the zebrafish as an alternative to mammalian experimental models.
Adult zebrafish were raised and maintained under internationally accepted conditions in the Institute of Molecular and Cell Biology (IMCB) Zebrafish Aquarium Facility, accredited by the Animal and Veterinary Authority (AVA) of Singapore. All experimental procedures were performed in compliance with and approved by the Agency for Science Technology and Research (A*STAR) Biological Resource Centre Institutional Animal Care and Use Committee (IACUC Project \#110638). Most experimentation and analysis was restricted to the first 5 days postfertilization (dpf). Homozygous mutant fish were regularly monitored, and any showing signs of distress were humanely euthanized following accepted protocols.

\section{Zebrafish strains and husbandry}

Adult fish were maintained on a 14 hour light/10 hour dark cycle at $28^{\circ} \mathrm{C}$ in the AVA (Singapore) certificated IMCB Zebrafish Facility. Previously described zebrafish strains used were: $\operatorname{Tg}(\operatorname{smyhc1}: G F P)^{i 104}$ [22]; $\operatorname{prdm}^{\text {nrd }}$ [23]; $\mathrm{Tg}$ (prdm1:GFP) ${ }^{i 106}$ [22] and $\operatorname{Tg}(\operatorname{actin} 1 \beta: G A L 4)^{i 269}$ line [24].

\section{Generation of UAS:Sox6-GFP}

The sox6 ORF was amplified by PCR and cloned into pDONR221 to make pME-sox6, and then recombined with p5E-UAS, p3E-GFP and pDestTol2pA by gateway cloning. The resultant UAS:sox6-GFP plasmid was injected into one-cell stage embryos with tol2 mRNA to generate the $\operatorname{Tg}(U A S \text { :sox6-GFP })^{i 295}$ line.

\section{Real-time PCR analysis}

Real-time PCR was performed on a Bio-Rad (Hercules, CA, USA) iQ5 real-time PCR detection system using KAPA SYBR FAST qPCR Kit (KAPA Biosystems, Wilmington, MA, USA), according to the manufacturer's protocols. Primer sets were designed for smyhc1 (forward, CCTGG TGTCTCAGTTGACCA; reverse, TGTGCCAGGGCAT TCTTT), tnnc1b (forward, GCAAGATCGACTACGAC GAG; reverse, AGGCAGCATTGGTTCAGG), mylz2 (forward, CAGGTTCACCGCAGAGGA; reverse, TTCGT TTTCTTGATTCCAAGG), and b-actin (forward, TGGC ATTGCTGACCGTATGC; reverse, GTCATGGACGCC CATTGTGA). Real-time PCR was performed with cDNA samples synthesized from $3 \mu \mathrm{g}$ of total RNA from approximately 50 embryos. Relative mRNA expression levels were calculated based on cycle threshold and amplification efficiency of each primer set. Expression of $b$-actin gene was used as internal control for normalization.

\section{Generation, selection and genotyping of sox 6 mutant alleles}

Plasmids encoding zinc-finger nucleases (ZFN) specific for the zebrafish sox6 gene were purchased from Sigma-Aldrich (St. Louis, MO, USA). The zinc-finger nuclease was designed so that it targeted the sequence CTGGCACGCCAAcagcaAGAGCAGGTGAGAATGTG, which is present in 
both isoforms of Sox6, upstream of the HMG box. Capped polyadenylated RNA from each plasmid was produced by in vitro transcription, and a range of doses was injected into one-cell stage zebrafish embryos.

G0 adults derived from embryos injected with ZFN capped RNA were incrossed and their progenies (G1) individually genotyped by $\mathrm{PCR}$ using the sox6 $\mathrm{ZF}$ forward primer (GGGTGCAGGGTTGTGAAGTG) and the sox6 ZF reverse primer (ATACATGCACATTACTG CAGGTG) followed by Sanger sequencing using the sox6 ZF seq primer (CTTCCTTCTTCCATTTTGTTC). Two alleles, $\operatorname{sox} 6^{i 291}$ and $\operatorname{sox} 6^{i 292}$, were isolated, each of which introduces a premature stop codon into the open reading frame that are predicted to encode truncated forms of the protein.

\section{Generation of tnnc1b:eGFP transgenic line}

An eGFP-SV40pA-FRT-Kn-FRT recombineering targeting cassette and red recombineering system in EL250 cells were used to insert eGFP with an SV40 polyadenylation site at the tnnc1b ATG start site in BAC ZC137P17 [25], which has at least $25 \mathrm{~kb}$ of upstream sequence and $200 \mathrm{~kb}$ of downstream sequences from the tnnclb gene. This BAC was further modified by the addition of Tol2 sites and a stable line, $\operatorname{Tg}(\text { BACtnnc1b:EFGP })^{i 293}$, was generated using Tol2-mediated transgenesis [26]. Deletion derivatives of the BAC were made by further targeted recombination events. Downstream deletions were made by targeting the iTol2-Amp-iTol2 cassette into the eGFP modified BAC but designing the homology arms so that the right arm had homology to the sequence $1 \mathrm{~kb}$ downstream from the tnnclb stop codon and the left arm had homology to the other side of the chloramphenicol marker resulting in the chloramphenicol gene being replaced by the ampicillin and a large amount of downstream sequence deleted from the BAC. Upstream deletions were made by targeting a construct containing a kanamycin resistance gene to different loci in the BAC so differing amounts of upstream sequence were recombined out of the BAC. Successful deletion of upstream and downstream sequences was confirmed by PCR. Smaller constructs containing the eGFP reporter sequence were PCR-amplified out of the BAC and cloned into the pDB739 vector that contains Tol2 sites [27] (gift of Steve Ekker, Mayo Clinic, Rochester, MO, USA).

A $\beta$-globin minimal promoter reporter vector was generated by excising $\beta$-globin-eGFP-polyA from $\beta$ g-eGFPSP72 [28] using EcoRI and cloned into the same site of pDB739 [27]. Potential enhancer fragments were cloned into this vector.

\section{Site-directed mutagenesis}

To mutate potential Sox6 binding sites in the smyhc1:GFP promoter, the promoter proximal to the NotI site in the $\operatorname{Tg}(\text { smyhc:GFP })^{i 104}$ construct [22] was subcloned. Five potential Sox6 binding sites were identified manually and mutated from AACAAT to AAAAAT using the Stratagene (La Jolla, CA, USA) Quickchange Multi SiteDirected Mutagenesis Kit. After mutagenesis the mutated promoter was subcloned back into the full smyhc1:GFP plasmid and stable transgenic lines were created.

To mutate potential Sox6 binding sites in the tnnc1b: GFP promoter, the sequence $2.5 \mathrm{~kb}$ upstream of the start site of $t n n c 1 b$, as well as the first intron, was analyzed manually to identify possible Sox6 binding sites. Sites were mutated by designing forward and reverse primers that had approximately 10 -bp homology to the sequence 5 ' of the potential Sox6 binding site and 30-bp homology to the sequence 3' of the Sox6 site, with a 4-bp mismatch (ACAAT mutated to AGGG) in the core sequence of the Sox6 binding site. After PCR using these primer pairs and iProof DNA polymerase (Biorad, Hercules, CA, USA), $1 \mu \mathrm{l}$ of DpnI restriction enzyme was added to the reaction to digest the methylated plasmid template. After a 2-hour incubation at $37^{\circ} \mathrm{C}$, the sample was PCR-purified (AxyPrep PCR clean-up kit Axygen, Union City, CA, USA) and transformed. Sequences were analyzed using Lasergene SeqMan (DNA STAR Madison, WI, USA). Successfully mutated plasmids were injected into zebrafish embryos, and stable transgenic lines were created for each construct.

\section{In situ hybridization and immunofluorescence}

In situ hybridization of whole embryos and cryosections was performed as previously described $[29,30]$. Fluorescent in situ hybridization utilized anti-DIG peroxidase and fluorescence substrate Cy5 tyramide signal amplification (TSA, Perkin Elmer, Waltham, MA, USA) and was performed according to the manufacturer's protocol. Probes used were made from plasmids smyhc1 [22], tnnc1b [21], myh7b [31], ryrla [32] and proxla [33]. mylz10, tpm2, tnnt1 and tnnila were cloned from zebrafish cDNA into a pGEM-T vector (Promega, Madison, WI, USA). Probes were made by linearizing and transcribing with the appropriate restriction enzyme and polymerase respectively. Images were captured using the Carl Zeiss (Oberkochen, Germany) AXIO Zeiss Imager $\mathrm{M} 2$ and the AXIO Vision 4.7.2 software. To analyze the effects of the misexpression of Sox6, embryos were collected from a UAS:sox6-GFP;actin:GAL4 incross and separated based on their Sox6-GFP expression; embryos expressing strong Sox6-GFP were collected into one tube, and embryos expressing no Sox6-GFP were separated into another tube. Reactions were carried out in separate tubes, with the same number of embryos in each and were stained for exactly the same length of time.

\section{Monitoring $\mathrm{Ca} 2+$ flux using GCaMP3}

The $2 \mathrm{dpf}$ larvae were mounted in $2 \%$ low-melting agarose gel containing $50 \mu \mathrm{M}$ blebbistatin, and muscle contraction 
was induced by $40 \mathrm{mM}$ pentylenetetrazole (PTZ) as previously described [34]. The smyhc1:GCaMP3 construct was generated by replacing the $g f p$ coding sequence of the 9.7kb smyhc1:gfp construct [22] with GCaMP3 coding sequence and used to generate a transgenic line, $\operatorname{Tg}($ smyhc1: $G C a M P 3)^{i 280}$. Fast-twitch specific transient transgenics were generated by injecting the mylz2:GCaMP3 construct [34]. A total of $1 \mathrm{nl}$ of DNA plasmid was injected into the one-cell stage embryo at $20 \mathrm{ng} / \mu \mathrm{l}$. Fluorescent change was measured every 70 to $90 \mathrm{~ms}$ with Olympus (Tokyo, Japan) FV-1000 Fluoview confocal microscopy, and average fluorescence intensity was analyzed using Olympus FV10-ASW software.

\section{Immunohistochemistry}

Antibody staining was performed as previously described $[22,31]$ at the following dilutions: mAb F310 (anti-fast myosin light chain; DSHB) at 1:50; mAb F59 (anti-slow myosin heavy chain; DSHB) at 1:50; Rabbit anti-eGFP (Torrey Pines) 1:500; and rabbit anti-zebrafish Sox6 [31] 1:500. The following secondary antibodies were used: anti-rabbit IgG-488 (Invitrogen) 1:100 and anti-mouse IgG-546 (Invitrogen) 1:1000. Specimens were imaged with an Olympus Fluoview confocal microscope. Images were acquired using Olympus FV10-ASW software and analyzed using ImageJ software (http://rsbweb.nih.gov/ij/).

\section{Results}

Misexpression of Sox6 in adaxial cells represses slow-twitch specific gene expression

To investigate whether Sox6 activity is sufficient to repress slow-twitch specific gene expression, we generated a transgenic line in which a Sox6-GFP fusion protein can be driven under the control of the GAL4 transcriptional activator. Fish carrying this transgene, $\mathrm{Tg}$ $(U A S: s o x 6-G F P)^{i 295}$, were crossed with those carrying a previously described muscle specific actin:GAL4 transgene [24]. Fluorescent in situ hybridization of embryos that were 30-hours postfertilization (hpf) indicated that expression of tnnclb and smyhc1 was reduced in the Sox6-GFP expressing fibers (Figure 1A-D). Similarly, expression of the slow-specific genes troponin 11 (tnnt1), troponin ila (tnnila), myosin light chain 10 (mylz10), tropomyosin 2 (tpm2) and the ryanodine receptor 1 a (ryrla) also appeared to be downregulated in embryos expressing Sox6-EGFP (Additional file 1: Figure S1). To confirm these findings, we performed qPCR analysis of smyhc1 and tnnc1b transcription in Sox6-GFP and control embryos. In both cases, we found a modest but statistically significant reduction in transcript levels; by contrast, levels of mylz2 transcript did not differ significantly between Sox6-GFP expressing and control embryos (Figure 1E). Embryos with strong GFP expression in the head muscle at $4 \mathrm{dpf}$ were sorted from GFP-negative embryos. In situ hybridization for tnnc1b transcripts revealed no effect of ectopic Sox6 activity on tnnc1b expression in the head muscles (Additional file 2: Figure S2). These findings are in line with the previous demonstration that ectopic expression of Sox6 can repress Prox1a expression in adaxial cells [21]. Taken together, they demonstrate that Sox6 activity is sufficient to inhibit the expression of slow-twitch muscle genes in the embryonic trunk muscle of the zebrafish.

\section{cis-acting sequences mediate Sox6 dependent repression of tnnc $1 b$ and smyhc 1}

Previous studies have identified cis-regulatory regions of the $s m y h c 1$ gene that drive reporter gene expression specifically in adaxial cells and slow-twitch fibers [22,35]. Five potential Sox6 sites were identified in this upstream sequence and mutated; transgenic lines carrying the mutated reporter construct showed ectopic expression of the reporter in fast-twitch fibers of embryos at $48 \mathrm{hpf}$ (Figure $1 \mathrm{G}$ and $\mathrm{H}$ ), implying that these sites are required for the lineage specific repression of smyhc1. The ectopic eGFP expression was not observed in every fast-twitch fiber but was restricted to a subset of fast-twitch fibers.

To explore the basis of $t n n c 1 b$ regulation further, an eGFP reporter cassette was inserted into a tnnc1b containing BAC by homologous recombination [25] such that the eGFP ATG start site replaced the tnnc1b ATG start site. The resulting tnnc1b:eGFP reporter gene recapitulated the endogenous pattern of tnnc1b expression in transgenic embryos, with expression restricted to the slow-twitch muscle and overlapping precisely with the endogenous tnnclb transcript (Figure $2 \mathrm{~A}$ and B). To define the cis-regulatory regions more precisely, several reporter constructs containing differing lengths of the $t n n c 1 b$ upstream and downstream sequence were generated and their activities assayed in transgenic embryos (Figure 2C). This deletion analysis revealed that the first intron of tnnc1b is essential for the expression of the gene. This putative intron 1 enhancer element was cloned downstream of a $\beta$-globin minimal promoter vector to assay its activity. Interestingly, eGFP expression was observed throughout the myotome in both the fast-twitch and slow-twitch fibers (Figure 2D and E) indicating that it acts as a general muscle-specific enhancer. Previous analyses identified a skeletal muscle enhancer in the first intron of the mouse Tnnc1 gene and this shows high conservation with the human Tnnc1 gene [36]. The first intron of human Tnnc1 was cloned into the $\beta$ globin minimal promoter vector and found to drive eGFP expression in both the slow-twitch and fast-twitch muscle in stable transgenic zebrafish embryos (Figure 2F). It follows that the slow-twitch specificity of the tnnclb gene is mediated by repressor elements upstream of its promoter. 


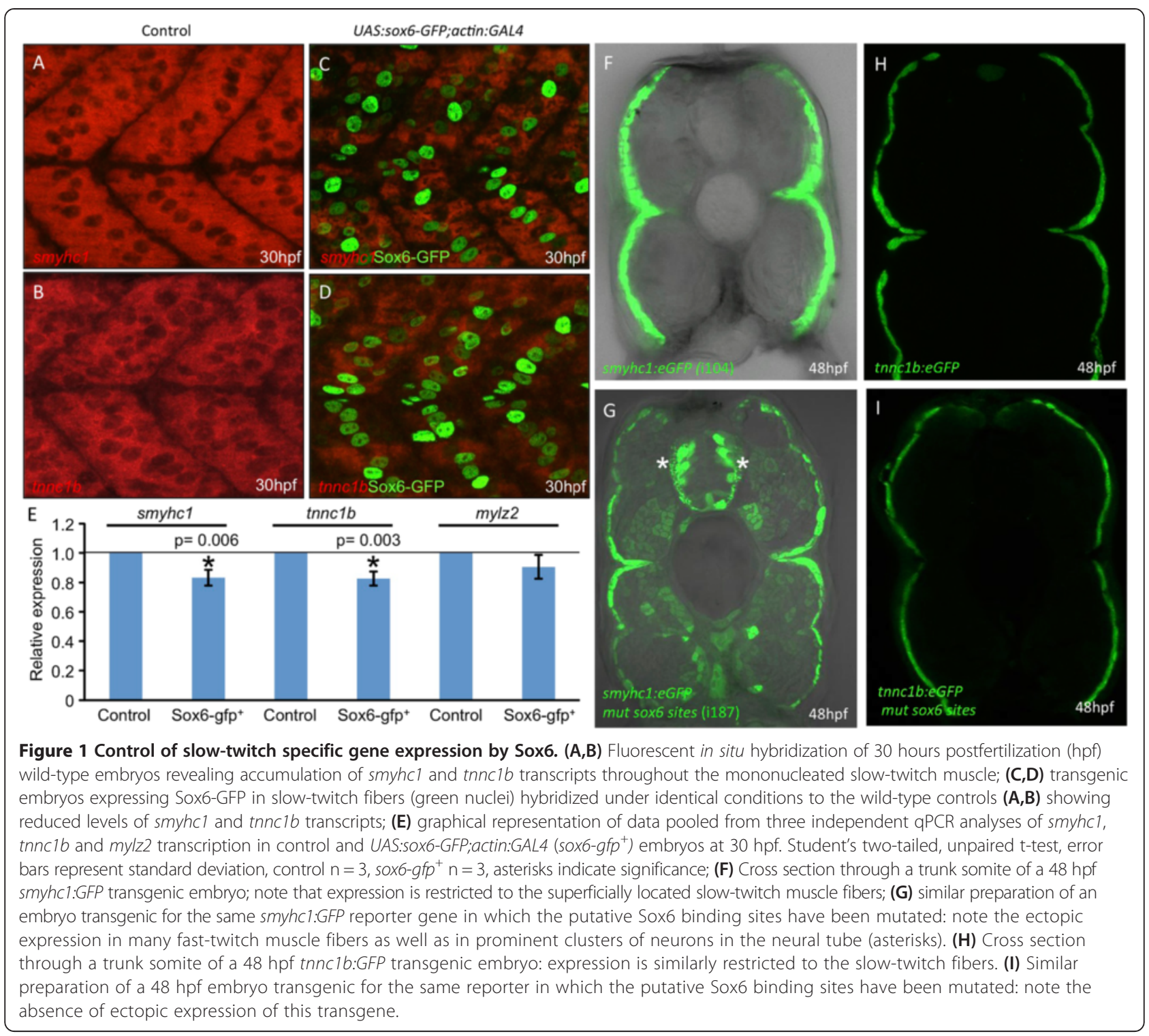

Five potential Sox6 binding sites were identified in the 2.5-kb upstream sequence + intron 1 . Progressive mutation of these sites had no effect on reporter gene expression in transgenic embryos (Figure 1I; Additional file 3: Figure S3). Further deletion of the $t n n c 1 b$ promoter revealed that just 270 bp upstream of the transcription start site together with the first intron was sufficient to drive slowspecific expression in zebrafish embryos (Figure 2C). This 270-bp upstream region contains no canonical Sox6 sites, though two sites with an imperfect match (0.85 identity) were detected. Potential sites for many other transcription factors were also identified by in silico analysis (data not shown), but none of these stood out as an obvious candidate for mediating slow-twitch fiber specific expression.

\section{Sox6 represses only a subset of slow-specific genes in}

\section{fast-twitch fibers}

Expression of sox 6 can first be detected in the somites at the ten-somite stage [31] when it is restricted to fasttwitch fiber progenitors and excluded from adaxial cells. To investigate Sox6 function further, mutant alleles were created using zinc-finger nuclease (ZFN) mediated targeted mutagenesis [37] (Figure 3A). Homozygous mutant embryos lacked full-length Sox6 protein in both fast-twitch fibers and in the forebrain, as assayed by whole-mount immunohistochemistry using a Sox6-specific antibody (Figure 3B-E).

In line with previous morpholino-based analyses [21], ectopic tnnc1b expression was observed throughout the fast-twitch domain in the trunk and tail of sox6 mutant 

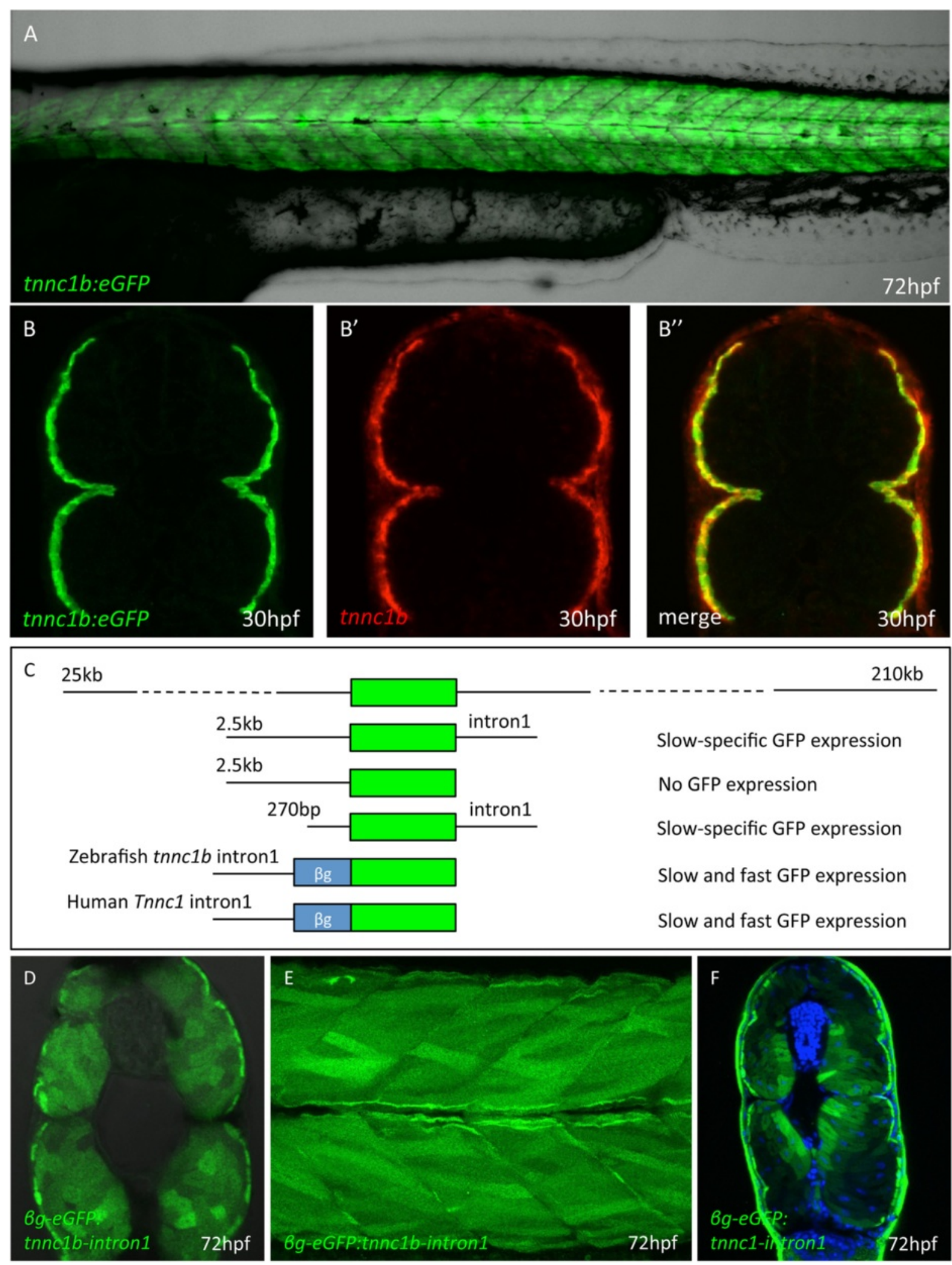

Figure $\mathbf{2}$ (See legend on next page.) 
(See figure on previous page.)

Figure 2 Defining the $\boldsymbol{t n n} \mathbf{1} \mathbf{b}$ cis regulatory region. (A) Trunk region of a Tg(BACtnncib:eGFP) ${ }^{\text {i293 }}$ larva 72 hpf showing reporter gene expression specifically in the slow-twitch muscle. (B) Cross section through the mid-trunk region of a 30 hpf Tg(BACtnncib:eGFP) (293 $^{2}$ embryo showing reporter gene expression restricted to the superficial layer of muscle fibers: ( $\left.\mathbf{B}^{\prime}\right)$ the same section showing endogenous tnnc $1 b$ mRNA assayed by fluorescent in situ hybridization. (B") Merged images showing that the reporter faithfully recapitulates the expression pattern of the endogenous gene. (C) Schematic representation of deletion derivatives of the BACtnnc1b:eGFP reporter construct (Green box=eGFP); the expression patterns observed in transgenic lines carrying these construct is indicated on the right. (D) Cross section and (E) optical sagittal section of a 72 hpf transgenic larva showing expression of eGFP throughout the myotome driven by the first intron of the zebrafish tnn 1 cb intron and a mammalian $\beta$ globin minimal promoter. (F) Cross section of a 72 hpf transgenic embryo in which expression of eGFP is driven by the first intron of the human slow troponin c gene. The strong signal in the skin in is a staining artefact and was not present in live embryos.

embryos from approximately $22 \mathrm{hpf}$ onward (Figure 4A and $\mathrm{C}$ ), but not in craniofacial fast-twitch muscles (Additional file 4: Figure S4). Similarly, the tnnc1b: eGFP transgene was ectopically expressed in the fasttwitch fibers in sox6 homozygotes (Figure $4 \mathrm{~B}$ and D). Like $t n n c 1 b$, the slow-specific ryrla (Figure $4 \mathrm{E}$ and I) and tnnt1 (Additional file 5: Figure S5A and F) were also ectopically expressed throughout the fast-twitch domain in sox6 mutants at $30 \mathrm{hpf}$. Interestingly, this was not the case for all slow-twitch muscle genes: the expression patterns of myh7b (Figure 4F and J) tnnila, mylz10, tpm2 and prox1a (Additional file 5: Figure S5B-E and G-J), were unaffected in sox6 mutant embryos at $30 \mathrm{hpf}$. Ectopic expression of smyhc1 could be detected in a small number of fast-twitch fibers by $30 \mathrm{hpf}$ in sox6 mutants, both by in situ hybridization for the endogenous transcript (Figure 4G and K) and by reporter gene expression (Figure $4 \mathrm{H}$ and $\mathrm{L}$ ). This pattern of ectopic expression resembled that of the mutant smyhc1:eGFP reporter construct described above (cf. Figure $1 \mathrm{H}$ ). By $3 \mathrm{dpf}$, fast fibers positive for tpm 2 and mylz10 expression could also be readily detected in sox6 mutant larvae (Figure 5B,C,E and F). Similarly, more fast fibers expressed smyhc1 in sox 6 mutants by 4 to $5 \mathrm{dpf}$ (Figure 5A,D,G and H).

In contrast to the aberrant expression of slow-specific genes, expression of fast myosin light chain proteins, detected with the F310 antibody (Figure 5 G' and H'), and expression of fast specific troponin subunit, assayed by WISH (Additional file 6: Figure S6), were unaffected in sox6 homozygotes.

\section{Loss of Sox6 rescues slow-specific gene expression in Prdm1 mutants}

Loss of Prdm1a function causes ectopic expression of sox6 in adaxial cells and concomitantly the loss or strong downregulation of slow-twitch muscle gene expression [13,21,22]; (Figure 6C and G). In the sox6;prdm1a double homozygotes, expression of smyhc1 in adaxial cells was partially restored (Figure 6D), while tnnc1b was uniformly expressed throughout the myotome (Figure $6 \mathrm{H}$ ). The migration of the slow-twitch progenitors is also disrupted in prdm1a mutants (Elworthy et al. [22]); this effect was not rescued in prdm1a;sox6 double mutants (YO and PWI, unpublished observations: see also Figure 6D).

\section{Loss of Sox6 results in altered physiology of fast fibers}

Prompted by the ectopic expression of the slow-twitch specific ryanodine receptor encoded by ryrla in fasttwitch fibers in sox6 mutant larvae, we analyzed $\mathrm{Ca} 2+$ flux in muscle fibers, using transgenes driving muscle specific expression of the fluorescent calcium sensor GCaMP3 [38,39]. Animals carrying the smyhc1:GCaMP3 transgene express the sensor specifically in superficial slow-twitch muscle fibers (Figure 7A and B) while the mylz2:GCaMP transgene is specifically expressed in fast-twitch fibers [34]. Pentylenetetrazole (PTZ) treatment of transgenic larvae induced a rapid increase in fluorescence intensity followed by a return to the baseline level, reflecting $\mathrm{Ca} 2+$ flux from the sarcoplasmic reticulum into the muscle fiber. We analyzed three components of this calcium response: 1) time to maximum fluorescence intensity, 2) decay time to $50 \%$ of maximum fluorescence intensity, and 3) fluorescence amplitude (Figure 7C).

In wild-type larvae, the response time of slow-twitch fibers was shorter than in fast-twitch fibers, while the change in amplitude was smaller (Figure 7D and E). Notably, the response time of sox6 mutant fast-twitch fibers was significantly shorter than in their wild-type counterparts, resembling more that of wild-type slowtwitch fibers, whereas the amplitude change was unaffected. The fluorescence decay time showed no significant difference between slow-twitch and fast-twitch fibers in both wild-type and sox6 mutant fibers, indicating that the recovery mechanism of calcium ion flux is similar in both types of fiber (data not shown).

\section{Loss of Sox6 disrupts muscle fiber type at adult stages}

While homozygous sox6 mutant embryos and larvae displayed no observable morphological phenotype, by one month of age they were significantly smaller than their siblings (Figure 8A) and exhibited a slight curvature of the spine, a phenotype that became more severe with age. The extent of the curvature varied between individuals: some fish exhibited only a slight kink, while others 
A

Fok I Zinc-finger DNA binding domain

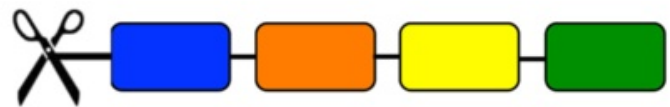

CTGGCACGCCAA_ragcaAGAGCAGGTGAGAATGTG GACCGTGCGGTTgtcgtTCTCGTCCACTCTTACAC

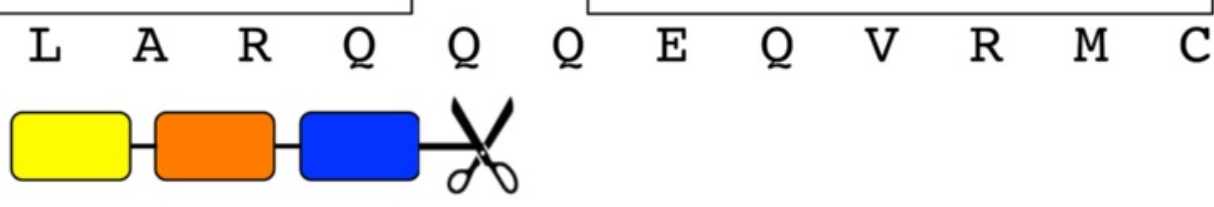

Zinc-finger DNA binding domain Fok I
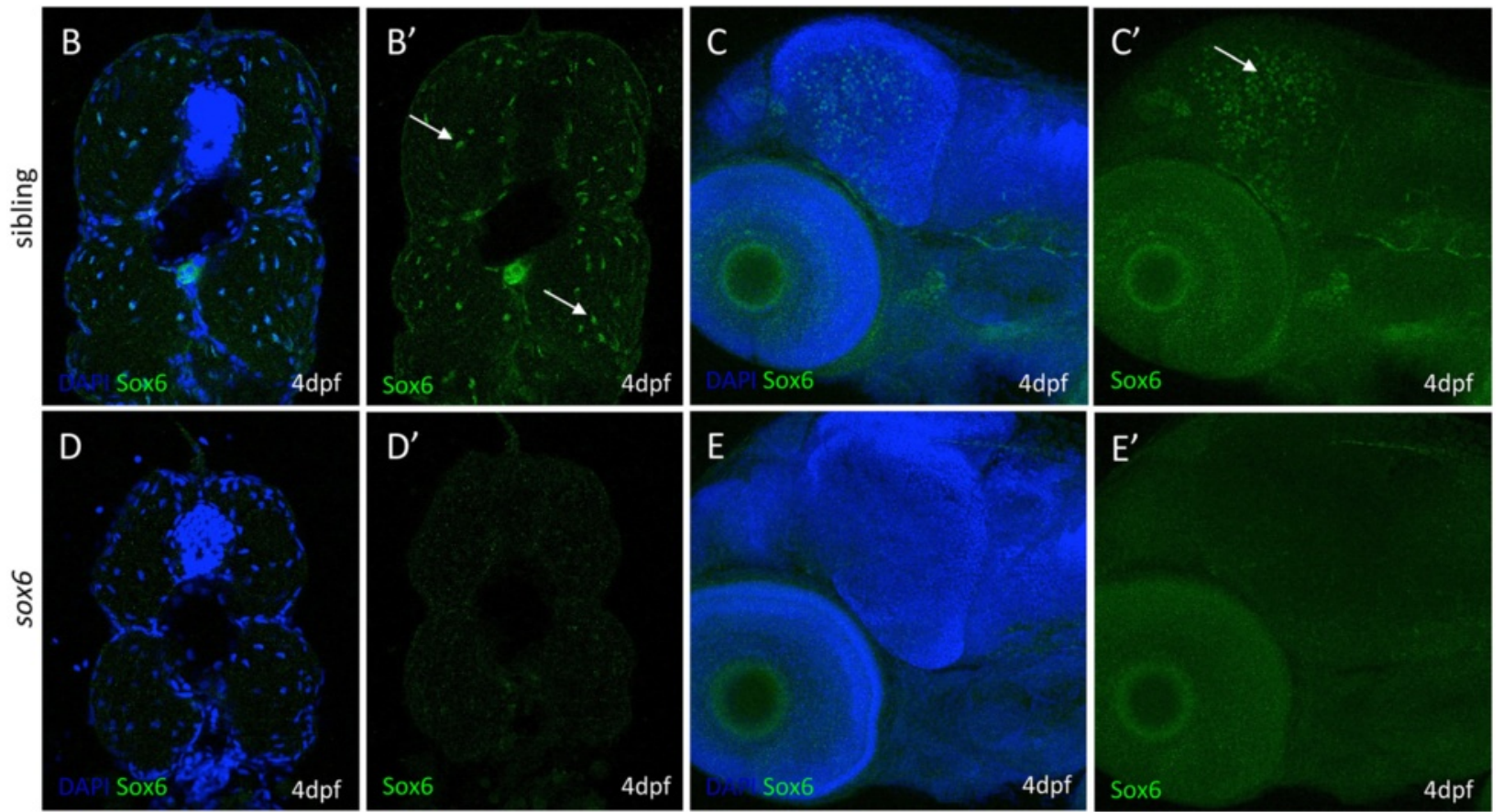

Figure 3 Targeted mutagenesis of the zebrafish sox6 locus. (A) Schematic representation of the zinc-finger nuclease designed to target genomic sequences in exon 8, upstream of the HMG box, of the zebrafish sox6 gene. (B-E) Immunohistochemical detection of Sox6 protein using a Sox6-specific antibody; Sox6 protein is detectable in the nuclei of fast muscle fibers (arrows) (B and $\mathbf{B}^{\prime}$ ) and the optic tectum (arrow) (C and $\mathbf{C}^{\prime}$ ) of wild-type embryos. In sox6 homozygous mutants, by contrast, no signal is detected in either the muscle (D and $\mathbf{D}^{\prime}$ ) or the optic tectum ( $\left(\mathbf{E}\right.$ and $\mathbf{E}^{\prime}$ ), indicating the successful generation of a null mutant. (Blue signal = DAPI).

displayed a massive crumpling of the spine. By $80 \mathrm{dpf}$, all surviving homozygous sox6 mutants displayed some degree of curvature as revealed by alizarin red staining (Figure 8B and C).

Around 10\% of homozygotes could survive to adulthood if raised in isolation from their siblings; these failed to breed, most likely due to the morphological abnormalities.
Adult muscle fibers were examined by visualizing the expression of the tnnc1b:eGFP and smyhc1:eGFP transgenes in cryostat sections through the trunk regions of sox $6 \mathrm{mu}-$ tants and their siblings. This revealed that the ectopic expression of tnnclb seen in mutant embryos and larvae persists into adulthood (Figure 9; cf. A and B). The level of expression of tnnc1b:eGFP in the fast domain, however, 

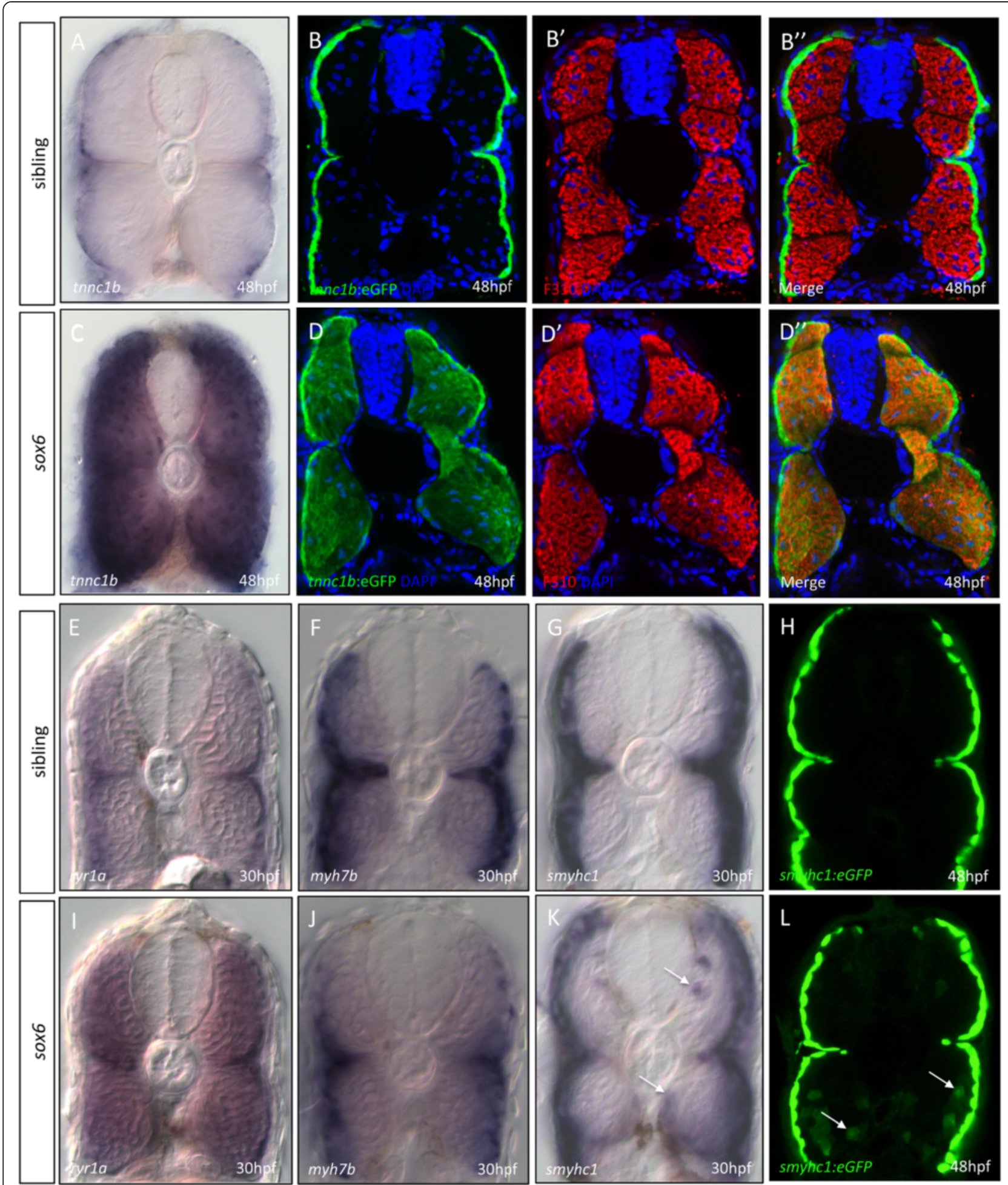

Figure 4 tnnc $1 b$ is ectopically expressed in fast-twitch fibers of homozygous sox 6 mutants. Endogenous tnnc $1 b$ expression (A) and tnnc $1 b$ : eGFP reporter expression (green) (B) is restricted to slow-twitch fibers of wild-type embryos at 48 hours postfertilization (hpf) and excluded from fast-twitch fibers ( $\mathbf{B}^{\prime}$ :red) as shown in merged image $\left(\mathbf{B}^{\prime \prime}\right)$. In sox6 mutants, endogenous tnncib $(\mathbf{C})$ and the tnncib:eGFP reporter $(\mathbf{D})$ are expressed in both slow and fast-twitch fibers ( $\mathbf{D}^{\prime}$ : red) as shown in merged image $\left(\mathbf{D}^{\prime \prime}\right)$. Expression of ryr 1 a is restricted to slow-twitch fibers in wild type (E) but ectopically expressed in the fast-twitch fibers in sox6 mutant embryos at $30 \mathrm{hpf}$ (I). By contrast, myh7b expression remains restricted to slow-twitch fibers in sox6 mutants (cf. $\mathbf{F}$ and $\mathbf{J}$ ). Slow-twitch specific expression of the endogenous smyhcl (G) and the smyhc1:GFP reporter (H) in wild-type embryos. In sox6 mutants (K,L), expression is largely similarly restricted but ectopically expressed in a few fast fibers (arrows). 

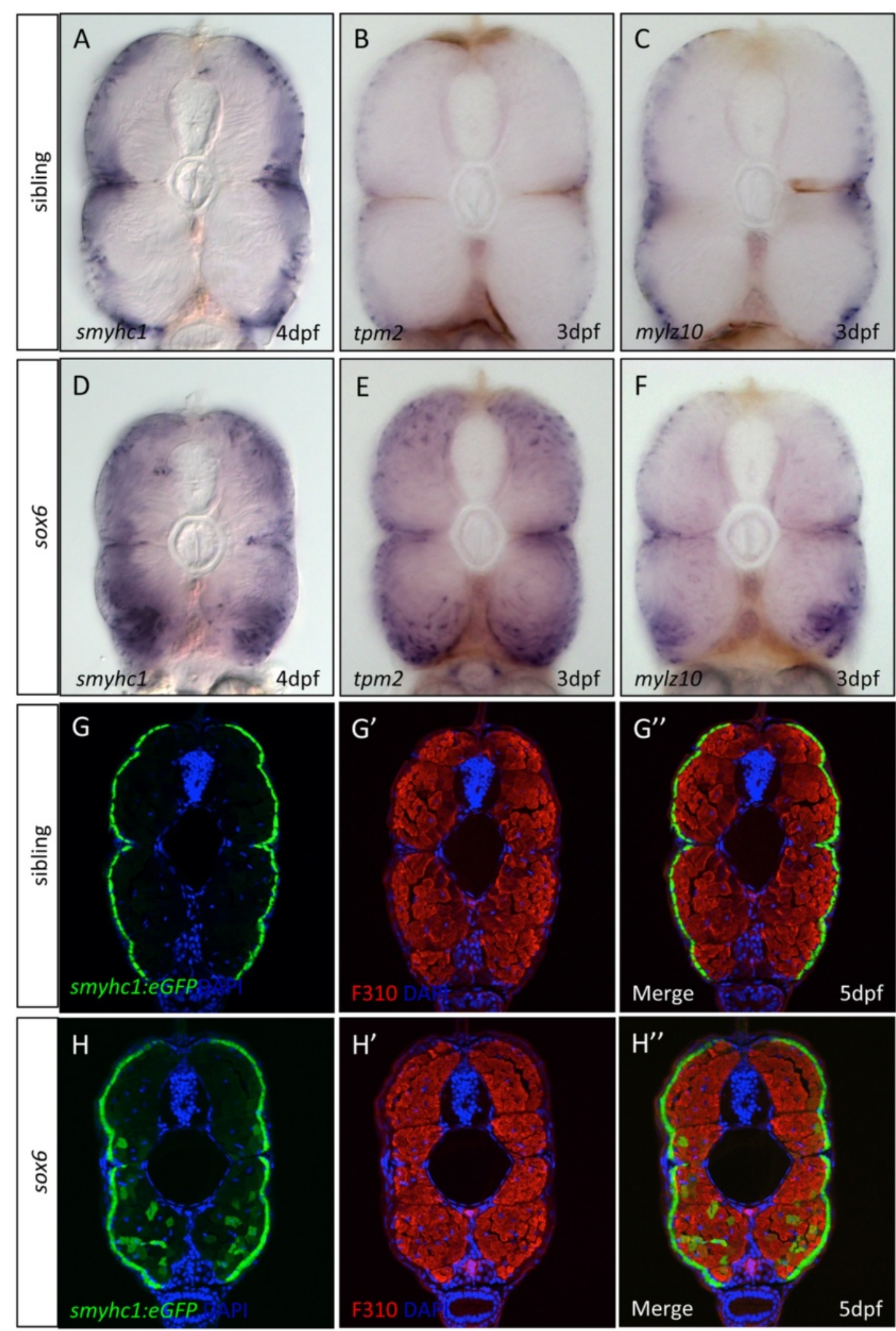

Figure 5 (See legend on next page.) 
(See figure on previous page.)

Figure 5 Partial de-repression of slow-twitch genes in sox6 mutant larvae. (A-C) smych1, tpm2 and mylz10 remain restricted to the slow-twitch fibers in wild-type larvae at 3 or 4 days postfertilization (dpf). (D-F) All three genes are ectopically expressed in some fast-twitch fibers in sox6 mutants by 3 or 4 dpf. (G-G") smyhc1:GFP expression (green) is excluded from fast-twitch fibers (red) in 5 dpf wild-type larvae. (H-H") In sox6 mutant larvae at 5 dpf, a subset of fast-twitch fibers ectopically express the reporter gene $\mathbf{( H )}$. The expression of the fast-twitch myosin revealed by mAb F310 (red) in the fast domain remains unaffected in sox6 mutants (cf $\mathbf{G}^{\prime}$ and $\mathbf{H}^{\prime}$ ).

was weaker than in the slow-twitch domain, resembling more that of intermediate fibers in wild type. Moreover, the morphology of the mutant myotome was altered compared to that of wild-type siblings, varying in shape and size between individuals. The wedge of eGFP-positive slow-twitch muscle, known as the lateralis superficialis $[7,22]$ extended more medially towards the midline of sox6 mutants (Figure 9; cf. A and B). In addition, the slowtwitch fibers were significantly smaller than in wild-type fish (Figure 9; cf. C and D).

By contrast, expression of smyhc1:eGFP remained restricted to the slow-twitch fibers in sox6 mutants. As in mutant embryos, sporadic ectopic expression of the smyhc1 reporter was observed in only a small number of fast-twitch fibers (data not shown). There was a slight medial expansion of the eGFP-positive domain, with some cells in the most medially located muscle fibers faintly expressing eGFP, as well as a dorsal and ventral expansion in the expression domain (Figure 9E and F).

\section{Discussion}

Previous studies have implicated the transcription factor Sox6 in the control of skeletal muscle fiber type identity both in mammals and fish [17-19,21]. In Sox6 mutant mice, various fast specific genes are downregulated in fast-twitch fibers while a number of slow fiber specific genes are upregulated; these include several myosin heavy chain genes as well as all three of the genes encoding the slow troponin subunits Tnnc1,Tnni1 and Tnnt1 [17-19]. By contrast, we observed only limited

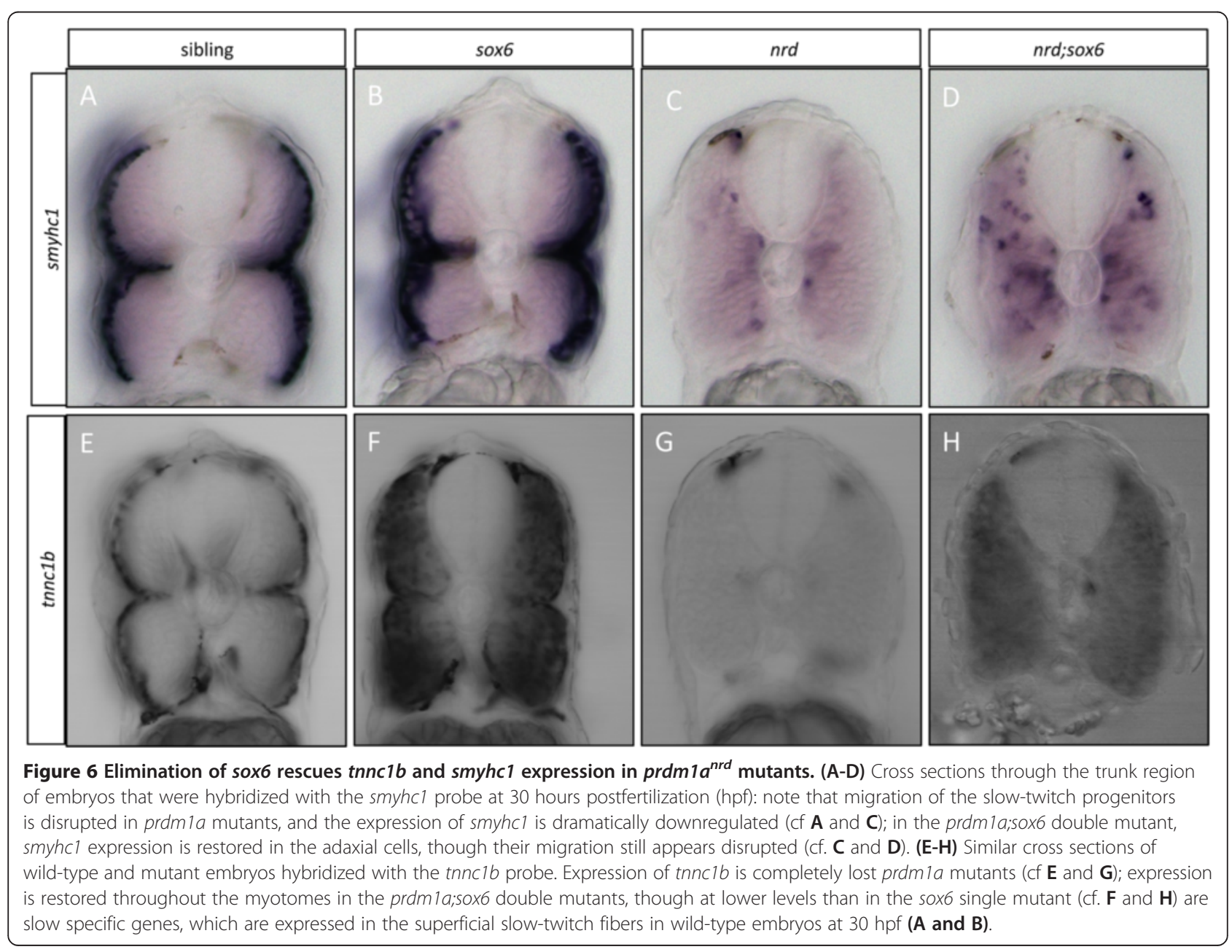



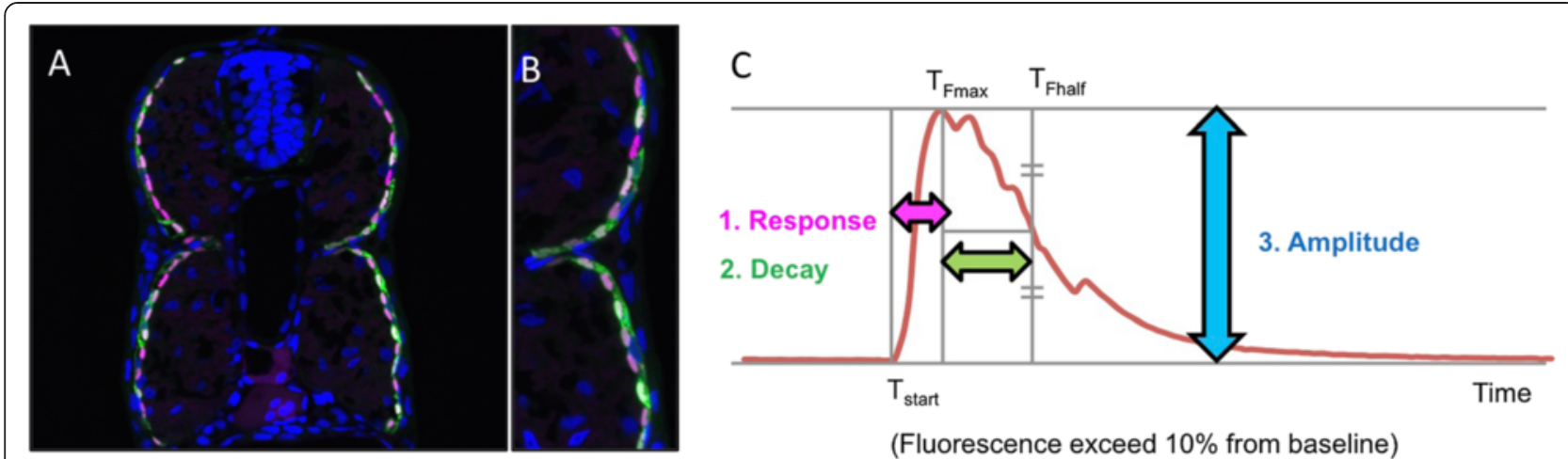

(Fluorescence exceed $10 \%$ from baseline)
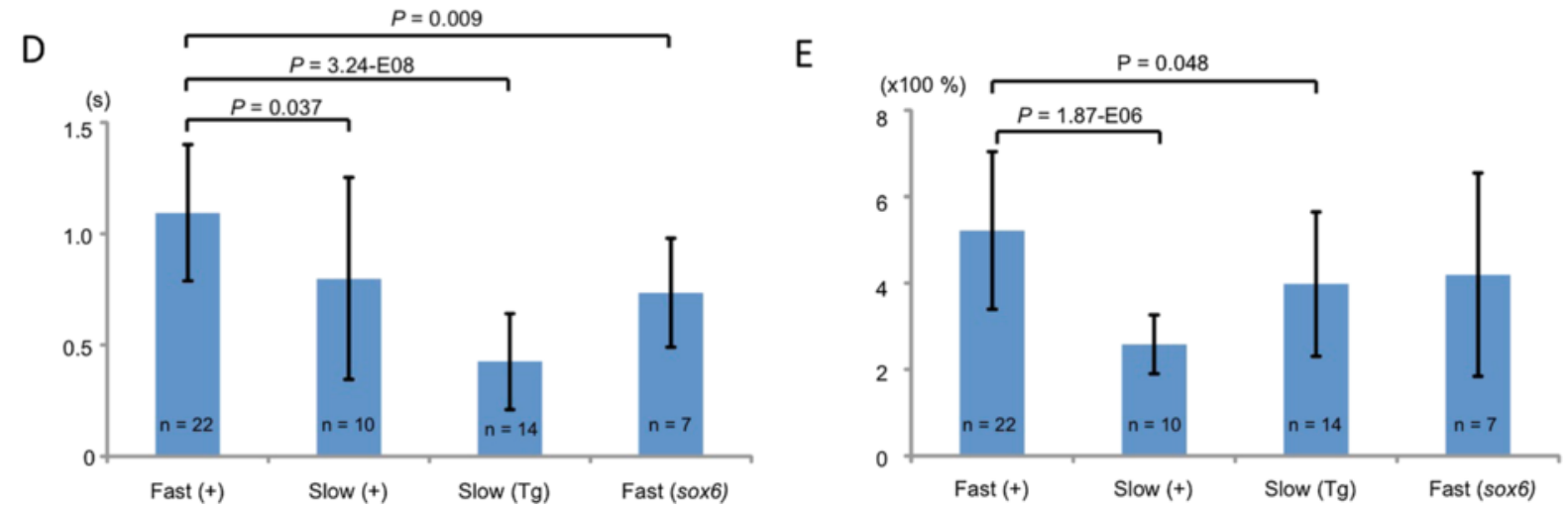

Figure 7 Sox6 controls the Ca2+ response of fast-twitch fibers. (A,B) Cross section through a trunk somite of a 48 hours postfertilization (hpf) Tg(smyhc1:GCaMP3) ${ }^{i 280}$ embryo, showing expression of GCaMP3 (green) is restricted to slow-twitch fibers, revealed by mAbF59 labeling (red). Nuclei are stained with DAPI (blue). (C) Stereotypical representation of the GCaMP3 signal in response to chemical stimulation indicating the three parameters measured in this analysis. (D) Response time in seconds (s) and (E) amplitude of Ca2+ response in fast and slow-twitch fibers of Tg (smyhc1:GCaMP3) ${ }^{i 280}(\mathrm{Tg})$ and transient transgenic wild-type (+) and sox6 mutant larvae (52 to 55 hpf); Student's two-tailed, unpaired t-test; Error bars represent standard deviation.

ectopic expression of smyhc1 at $30 \mathrm{hpf}$, with mylz10 and tmp 2 also showing limited ectopic expression in the fast fibers by $3 \mathrm{dpf}$.

On the other hand, ectopic Sox6 activity was sufficient to reduce expression all of these slow-twitch specific genes in the adaxial cells and consistent with this, we found that mutation of the putative Sox binding sites in the smyhc1 cis regulatory fragment resulted in ectopic expression of a GFP reporter gene in fast-twitch fibers.

One possible explanation for these paradoxical findings could be the presence of a paralogous sox 6 gene in the zebrafish genome, a consequence of the additional round of genome duplication that has occurred in teleosts [40]. Indeed, such teleost-specific sox6 paralogs were first described in puffer fish [41] and subsequent genome sequence analyses have revealed similar sox6 duplicates in Medaka and stickleback. Surprisingly, however, the zebrafish genome lacks the sox6a locus found in other teleosts (Additional file 7: Figure S7), thus ruling out this redundancy hypothesis.
Another potential explanation could be a partial functional overlap between Sox 6 and the closely related Sox 5 protein; indeed it is well established that Sox 5 and Sox6 function redundantly in a number of contexts in mammals [42-44]. To address this possibility, we generated sox5 null alleles using zinc finger nucleases (SE and PWI, unpublished data) and made compound homozygotes; however, we found no evidence of de-repression of additional slow-twitch genes in the double mutants (Additional file 8: Figure S8).

Nevertheless, the fact that ectopic Sox6 can repress expression of all of the slow-twitch genes we have assayed implies that some other Sox protein may act in parallel to Sox6 to regulate fiber type identity. There is as yet, however, no obvious candidate for such an additional Sox family member involved in myogenesis. A further puzzle is presented by our analysis of the tnnc1b promoter; this identified an upstream fragment sufficient to drive reporter gene expression specifically in slowtwitch fibers, which like the smyhc1 upstream regulatory 

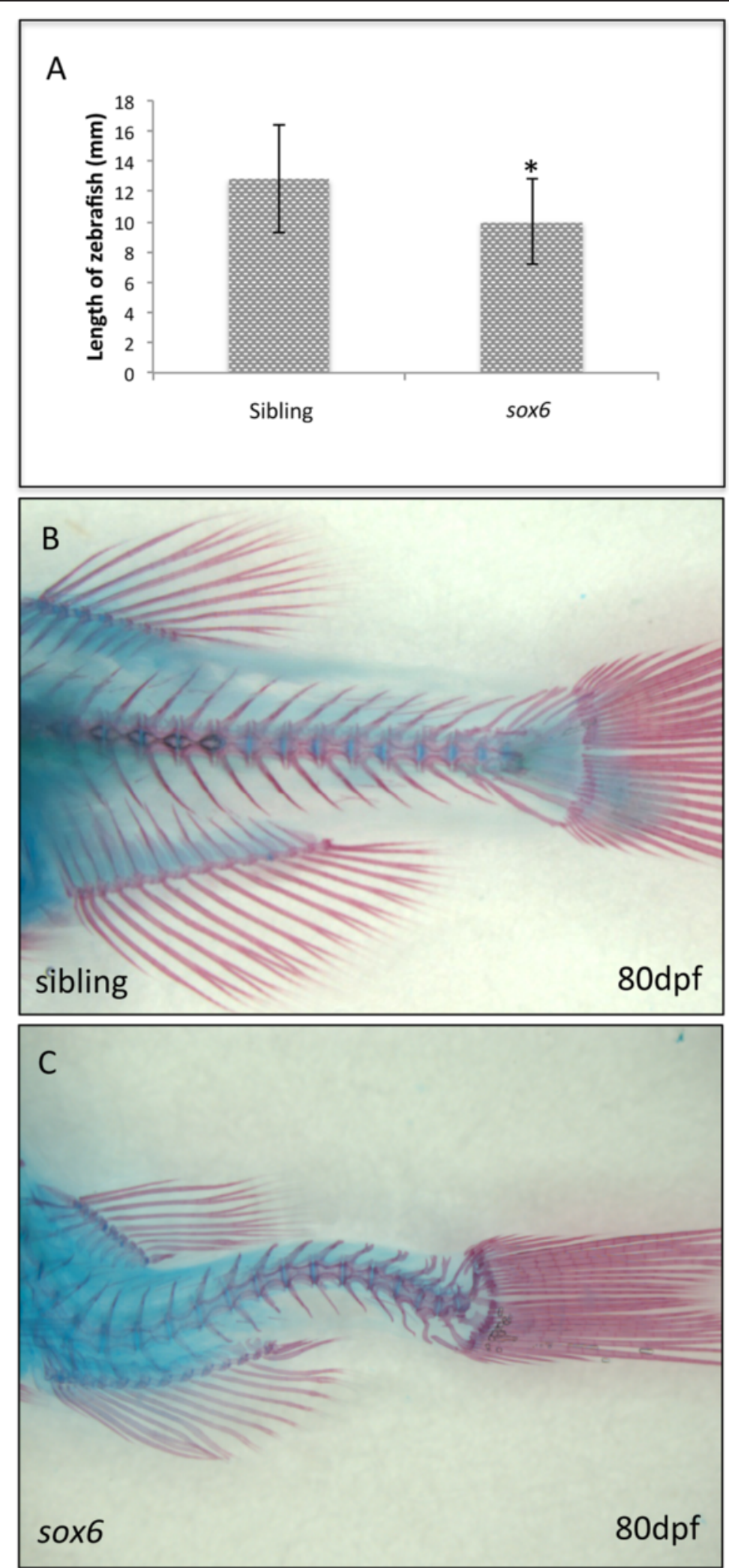

Figure 8 sox6 mutant adults are small and have spinal deformities. (A) sox6 mutant fish are significantly smaller than siblings by 1 month. Student's two-tailed, unpaired t-test $P<0.05$, error bars represent standard deviation, sibling $n=17$, mutant $n=17$, asterisk indicates significance. (B and C) By 80 dpf, there is clear curvature of the spine in all surviving homozygous sox6 mutant fish (C) as compared to siblings (B). 

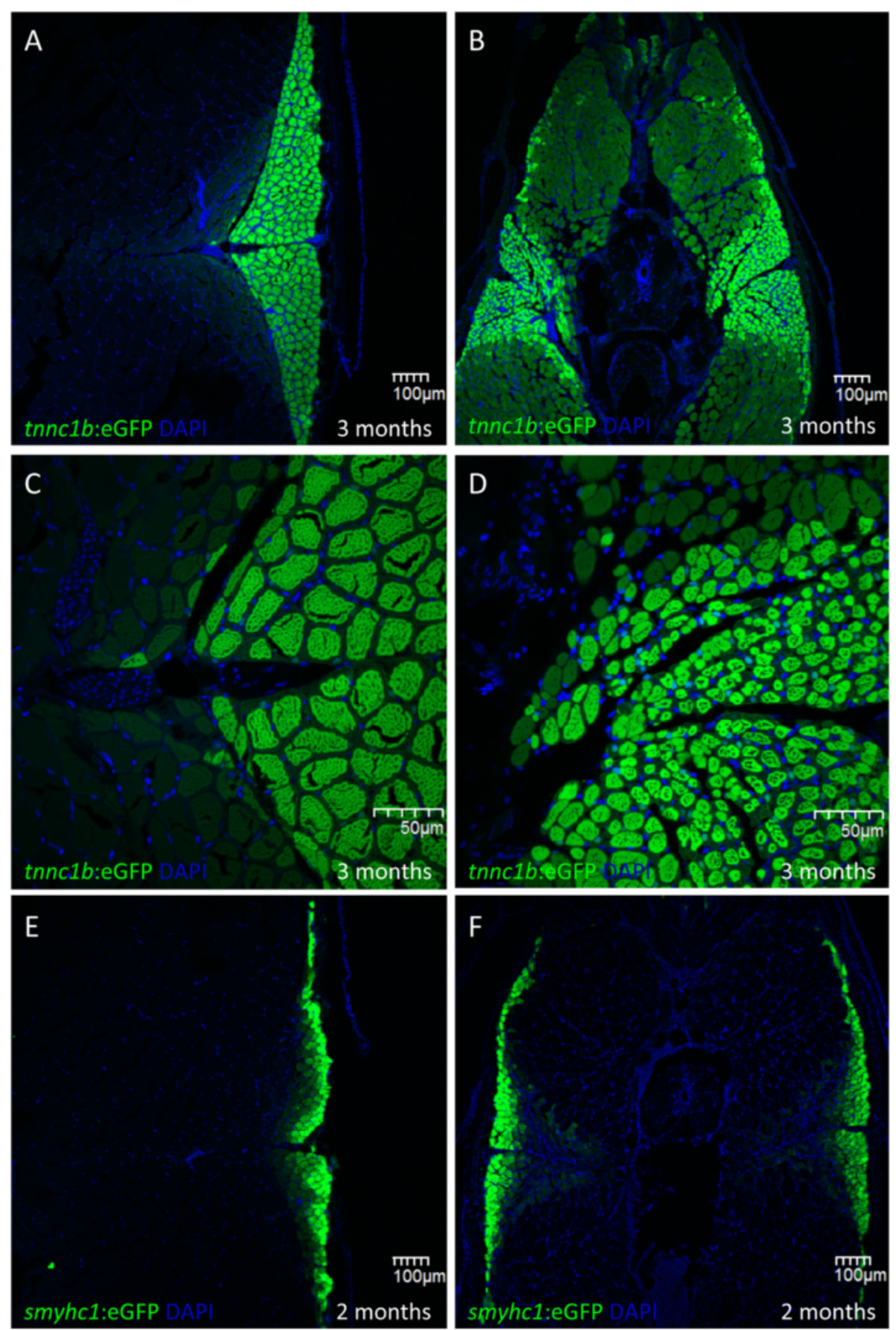

Figure 9 Disruption of fiber type identity in adult sox6 mutant fish. $(\mathbf{A}, \mathbf{B})$ at 3 months, expression of the tnncib:eGFP reporter is restricted to the medio-lateral wedge of slow-twitch fibers in wild-type fish. (C,D) In sox6 mutants, the reporter gene is also expressed at lower levels in fast-twitch fibers while the slow-twitch fibers extend closer to the midline. The diameter of the slow-twitch fibers is significantly reduced compared to wild-type (Cf. B and D). (E) The smyhc1:GFP reporter is expressed at high levels in the slow-twitch fiber of 2-month-old fish. (F) the reporter shows a similar pattern of expression in sox6 mutants at the same stage, with lower level expression extending more medially than in wild type.

element described previously, contains multiple putative Sox6 binding sites. Surprisingly, however, mutation of these sites failed to cause ectopic reporter gene expression in fast-twitch fibers. Moreover, partial deletion of this fragment identified a minimal upstream element devoid of canonical Sox6 binding sites that retains slow-specific enhancer activity. It is of course possible that other cryptic Sox6 binding sites are present in this fragment or that Sox6 binds via interaction with another DNA binding protein. In the absence of a Sox6 antibody that can be 
used in chromatin precipitation assays, we have been unable to address the interaction between Sox6 and this element directly.

The homeodomain transcription factors Six $1 \mathrm{a}$ and $\mathrm{Pbx}$ have previously been implicated in the transcriptional activation of fast-specific genes in zebrafish $[45,46]$. Since both proteins can also act as transcriptional repressors, it seems possible that they might act in concert with Sox6 to repress the expression of slow-specific genes in the fast-twitch fibers muscle. Morpholino-mediated knockdown of either gene in sox6 mutant embryos, however, had no discernible effect on the expression of the smyhc1: $g f p$ transgene (data not shown).

In any case, it is clear from our analysis that Sox6 is not the sole mediator of slow-twitch gene repression in fast-twitch fibers. In this respect, it is worth noting that neither Ppargcla (PGC-1a) nor Sdha, both of which are associated with the oxidative metabolism characteristic of slow-twitch fibers, are upregulated in fast-twitch fibers of mouse Sox6 mutants $[19,20]$. Nevertheless, the disparity between mammals and zebrafish in the selective repression of sarcomeric protein genes in the latter but not the former is striking and worthy of further investigation.

Using fiber type-specific expression of the $\mathrm{Ca} 2+$ reporter GCaMP3, we found that fast- and slow-twitch fibers show different calcium responses and that loss of Sox6 function modified the fast-twitch fiber specific response. In a previous study, the calcium indicator Calcium Green-1 dextran was used to analyze $\mathrm{Ca} 2+$ transients in wild-type and mutant larvae [32]. The responses reported for slow and fast-twitch muscles in wild-type embryos appeared quite similar, although no statistical analysis was performed to confirm this. The discrepancy between these earlier results and our findings could possibly be ascribed to the different mode of muscle stimulation - mechanosensory stimulation versus drug-induced convulsion - used in the two studies. Nevertheless, it is notable that loss of Sox6 function modified the response of the fast-twitch fibers in our analysis. We did not, however, observe a significant change in the half decay time of the Ca2+ signal in sox6 mutant embryos. On the other hand, it was reported that decay time is delayed in the atp2a1 mutant that encodes ATPase Ca2+ pump SERCA1 [47]. This suggests that expression of SERCA1 is independent of Sox 6 function.

In contrast to the perinatal lethality of the mouse Sox6 mutation, a small proportion (approximately $10 \%$ ) of zebrafish homozygous for the sox6 null allele survived to adulthood. These fish showed persistent ectopic expression of the $t n n c 1 b$ gene throughout the fast-twitch fibers indicating a continuous requirement for Sox6 in the maintenance of fiber type identity. In addition, the muscle blocks were misshapen and reduced in size, while slowtwitch fibers appeared significantly smaller than in wild type. The mutant fish also exhibited a marked scoliosis; while this may be a secondary consequence of the defects in the skeletal muscle, it could also reflect a direct requirement for Sox6 in the vertebral column. In this regard, it is notable that previous studies in mouse have revealed a role for both Sox 5 and Sox 6 in the formation of the extracellular matrix sheath of the notochord and of the nucleus pulposus, the gelatinous central portion of the intervertebral discs [48]. Further analysis is required to determine whether the scoliosis in sox6 mutant fish is a reflection of such a requirement.

\section{Conclusions}

Our analysis has established that the Sox6 transcription factor is a key regulator of fast-twitch muscle fiber differentiation in the zebrafish, a role similar to that ascribed to its murine ortholog. In the absence of Sox6 function, genes encoding slow-twitch specific sarcomeric proteins are ectopically expressed in fast-twitch fibers, the physiological properties of which are correspondingly shifted towards those of slow-twitch fibers. Not all slow-twitch specific genes are de-repressed in the absence of Sox6 function, however, which implies that additional factors act to suppress the slow-twitch differentiation program in fast-twitch fibers.

\section{Additional files}

\begin{abstract}
Additional file 1: Figure S1. The expression of slow-specific genes is downregulated in actin:GAL4; UAS:sox6-GFP embryos. The slow-twitch specific expression of tnnt1 (A), tnni1a (C), mylz10 (E), tpm2 (G) and ryr1a $(\mathrm{I})$ is downregulated in slow muscle fibers ectopically expressing Sox6-GFP $(B, D, F, H, J)$.
\end{abstract}

Additional file 2: Figure S2. Misexpression of Sox6 has no effect on the expression of tnnclb in the head muscles. (A-C) tnnclb expression in the head muscles of $3 \mathrm{dpf}$ wild type embryos. (D-F) tnnc 16 expression in embryos expressing Sox6-GFP in head muscles. There appears to be no difference in the expression of tnnc1b between wild type and actin:GAL4: UAS:SOX6-GFP fish.

Additional file 3: Figure S3. GFP expression is unaffected by mutation of potential Sox 6 binding sites in the $+2.5 \mathrm{~kb}+$ intron 1 tnnc1b:eGFP reporter. Expression remains restricted to the slow-twitch domain following mutation of one or more putative Sox6 binding sites (A-F).

Additional file 4: Figure S4. In situ hybridization for tnnclb mRNA reveals no difference in expression between wild type and sox6 mutants in the craniofacial muscles.

Additional file 5: Figure S5. Slow-specific muscle genes show a variable response to mutation of sox6. Like tnnc $1 b$ and ryr $1 a$, tnnt 1 is ectopically expressed throughout the fast domain of sox6 homozygous mutant embryos at $30 \mathrm{hpf}$ ( $\mathrm{A}$ and F). Conversely prox $1 \mathrm{a}$ expression is unaffected in sox6 mutant embryos at 22 hpf when transcript levels can be detected ( $\mathrm{B}$ and $\mathrm{G}$ ). Expression of tnnila ( $\mathrm{C}$ and $\mathrm{H}$ ), mylz10 ( $\mathrm{D}$ and $\mathrm{I}$ ) and tpm2 (E and J) is unaffected at $30 \mathrm{hpf}$ in sox6 mutants.

Additional file 6: Figure S6. Expression of fast specific troponin subunit genes in wild type and sox6 mutant embryos.

Additional file 7: Figure S7. Chromosomal location of sox6 genes in different fish species and $H$. sapiens. Two sox6 paralogs are present in the genomes of Fugu, stickleback and Medaka. The sox6B locus in each of these species is located in a chromosomal region that displays conserved 
synteny with the locations of the zebrafish and human sox6 gene. The sox6A genes of Fugu and stickleback are also located in a region of conserved synteny. This conservation has been lost in zebrafish along with the sox6A paralog (indicated by "?").

Additional file 8: Figure S8. Expression of $s m y h c 1$, myh7b and tnnc $1 b$ in wild type, sox5, sox6 and sox5; sox6 double mutants.

\section{Abbreviations}

dpf: days postfertilization; Hh: hedgehog; hpf: hours postfertilization; MPs: muscle pioneers; MRFs: myogenic regulatory factors; PTZ: pentylenetetrazole; SSF: superficial slow-twitch fibers; ZFN: zinc-finger nucleases.

\section{Competing interests}

The authors declare that they have no competing interests.

\section{Authors' contributions}

HEJ performed the analysis of the troponin C cis-regulatory region as well as the analysis of gene expression in wild type and mutant embryos and drafted the manuscript. YO generated the GCaMP3 transgenic line and performed the analysis of $\mathrm{Ca}^{2+}$ transients. WX performed the targeted mutagenesis of sox6 and generated the UAS:sox6-eGFP transgenic line. Stone Elworthy performed the analysis of the smyhc1 promoter. PWI and VTC conceived the study, participated in the experimental design and data analysis and finalized the manuscript. All authors read and approved the final manuscript.

\section{Acknowledgements}

This research was supported by the Singapore Agency for Science, Technology and Research (A*STAR). We are grateful to Niah Weixin for technical assistance.

\section{Author details}

${ }^{1} A^{*}$ STAR Institute of Molecular and Cell Biology, Proteos, 61 Biopolis Drive, Singapore 138673, Republic of Singapore. ${ }^{2}$ Bateson Centre, University of Sheffield, Western Bank, Sheffield S10 2TN, UK. ${ }^{3}$ Lee Kong Chian School of Medicine, Nanyang Technological University, Proteos, 61 Biopolis Drive, Singapore 138673, Republic of Singapore. ${ }^{4}$ Department of Medicine, Imperial College, South Kensington Campus, London SW7 2AZ, UK.

Received: 22 September 2014 Accepted: 10 December 2014 Published online: 27 January 2015

\section{References}

1. Schiaffino S, Reggiani C: Fiber types in mammalian skeletal muscles. Physiol Rev 2011, 91:1447-1531.

2. Jackson HE, Ingham PW: Control of muscle fibre-type diversity during embryonic development: the zebrafish paradigm. Mech Dev 2013, 130:447-457.

3. Weinberg ES, Allende ML, Kelly CS, Abdelhamid A, Murakami T, Andermann P, Doerre OG, Grunwald DJ, Riggleman B: Developmental regulation of zebrafish MyoD in wild-type, no tail and spadetail embryos. Development 1996, 122:271-280.

4. Blagden CS, Currie PD, Ingham PW, Hughes SM: Notochord induction of zebrafish slow muscle mediated by Sonic hedgehog. Genes Dev 1997, 11:2163-2175

5. Coutelle O, Blagden CS, Hampson R, Halai C, Rigby PW, Hughes SM: Hedgehog signalling is required for maintenance of myf5 and myoD expression and timely terminal differentiation in zebrafish adaxial myogenesis. Dev Biol 2001, 236:136-150.

6. Hammond CL, Hinits Y, Osborn DP, Minchin JE, Tettamanti G, Hughes SM: Signals and myogenic regulatory factors restrict pax3 and pax7 expression to dermomyotome-like tissue in zebrafish. Dev Biol 2007, 302:504-521.

7. Devoto SH, Melançon E, Eisen JS, Westerfield M: Identification of separate slow and fast muscle precursor cells in vivo, prior to somite formation. Development 1996, 122:3371-3380.

8. Barresi MJ, Stickney HL, Devoto SH: The zebrafish slow-muscle-omitted gene product is required for Hedgehog signal transduction and the development of slow muscle identity. Development 2000, 127:2189-2199.

9. Baxendale S, Davison C, Muxworthy C, Wolff C, Ingham PW, Roy S: The B-cell maturation factor Blimp-1 specifies vertebrate slow-twitch muscle fiber identity in response to Hedgehog signaling. Nat Genet 2004, 36:88-93.

10. Du SJ, Devoto SH, Westerfield M, Moon RT: Positive and negative regulation of muscle cell identity by members of the hedgehog and TGF-beta gene families. J Cell Biol 1997, 139:145-156.

11. Hirsinger $E$, Stellabotte F, Devoto $\mathrm{SH}$, Westerfield M: Hedgehog signaling is required for commitment but not initial induction of slow muscle precursors. Dev Biol 2004, 275:143-157.

12. Lewis KE, Currie PD, Roy S, Schauerte H, Haffter P, Ingham PW: Control of muscle cell-type specification in the zebrafish embryo by Hedgehog signalling. Dev Biol 1999, 216:469-480.

13. Roy S, Wolff C, Ingham PW: The u-boot mutation identifies a Hedgehogregulated myogenic switch for fiber-type diversification in the zebrafish embryo. Genes Dev 2001, 15:1563-1576.

14. Felsenfeld AL, Curry M, Kimmel CB: The fub-1 mutation blocks initial myofibril formation in zebrafish muscle pioneer cells. Dev Biol 1991, 148:23-30.

15. Hatta K, Bremiller R, Westerfield M, Kimmel CB: Diversity of expression of engrailed-like antigens in zebrafish. Development 1991, 112:821-832.

16. Henry CA, Amacher SL: Zebrafish slow muscle cell migration induces a wave of fast muscle morphogenesis. Dev Cell 2004, 7:917-923.

17. Hagiwara N, Ma B, Ly A: Slow and fast fiber isoform gene expression is systematically altered in skeletal muscle of the Sox6 mutant, $\mathrm{p} 100 \mathrm{H}$. Dev Dyn 2005, 234:301-311.

18. Hagiwara N, Yeh M, Liu A: Sox6 is required for normal fiber type differentiation of fetal skeletal muscle in mice. Dev Dyn 2007 236:2062-2076

19. An Cl, Dong Y, Hagiwara N: Genome-wide mapping of Sox 6 binding sites in skeletal muscle reveals both direct and indirect regulation of muscle terminal differentiation by Sox6. BMC Dev Biol 2011, 11:59.

20. Quiat D, Voelker KA, Pei J, Grishin NV, Grange RW, Bassel-Duby R, Olson EN: Concerted regulation of myofiber-specific gene expression and muscle performance by the transcriptional repressor Sox6. Proc Natl Acad Sci U S A 2011, 108:10196-10201.

21. von Hofsten J, Elworthy S, Gilchrist MJ, Smith JC, Wardle FC, Ingham PW: Prdm1- and Sox6-mediated transcriptional repression specifies muscle fibre type in the zebrafish embryo. EMBO Rep 2008, 9:683-689.

22. Elworthy S, Hargrave M, Knight R, Mebus K, Ingham PW: Expression of multiple slow myosin heavy chain genes reveals a diversity of zebrafish slow twitch muscle fibres with differing requirements for Hedgehog and Prdm1 activity. Development 2008, 135:2115-2126.

23. Hernandez-Lagunas L, Choi IF, Kaji T, Simpson P, Hershey C, Zhou Y, Zon L, Mercola M, Artinger KB: Zebrafish narrowminded disrupts the transcription factor prdm 1 and is required for neural crest and sensory neuron specification. Dev Biol 2005, 278:347-357.

24. Maurya AK, Tan H, Souren M, Wang X, Wittbrodt J, Ingham PW: Integration of Hedgehog and BMP signalling by the engrailed2a gene in the zebrafish myotome. Development 2011, 138:755-765.

25. Lee EC, Yu D, Martinez de Velasco J, Tessarollo L, Swing DA, Court DL, Jenkins NA, Copeland NG: A highly efficient Escherichia coli-based chromosome engineering system adapted for recombinogenic targeting and subcloning of BAC DNA. Genomics 2001, 73:56-65.

26. Kawakami $\mathrm{K}$ : Transgenesis and gene trap methods in zebrafish by using the Tol2 transposable element. Methods Cell Biol 2004, 77:201-222.

27. Balciunas D, Wangensteen KJ, Wilber A, Bell J, Geurts A, Sivasubbu S, Wang X, Hackett PB, Largaespada DA, Mclvor RS, Ekker SC: Harnessing a high cargo-capacity transposon for genetic applications in vertebrates. PLOS Genet 2006, 2:e169.

28. MacDonald RB, Debiais-Thibaud M, Talbot JC, Ekker M: The relationship between dlx and gad1 expression indicates highly conserved genetic pathways in the zebrafish forebrain. Dev Dyn 2010, 239:2298-2306.

29. Braissant $O$, Wahli W: Differential expression of peroxisome proliferatoractivated receptor-alpha, -beta, and -gamma during rat embryonic development. Endocrinology 1998, 139:2748-2754.

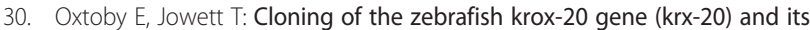
expression during hindbrain development. Nucleic Acids Res 1993, 21:1087-1095

31. Wang X, Ono Y, Tan SC, Chai RJ, Parkin C, Ingham PW: Prdm1a and miR-499 act sequentially to restrict Sox 6 activity to the fast-twitch muscle lineage in the zebrafish embryo. Development 2011, 138:4399-4404.

32. Hirata H, Watanabe T, Hatakeyama J, Sprague SM, Saint-Amant L, Nagashima A, Cui WW, Zhou W, Kuwada JY: Zebrafish relatively relaxed mutants have 
a ryanodine receptor defect, show slow swimming and provide a model of multi-minicore disease. Development 2007, 134:2771-2781.

33. Glasgow E, Tomarev SI: Restricted expression of the homeobox gene prox 1 in developing zebrafish. Mech Dev 1998, 76:175-178.

34. Baxendale S, Holdsworth CJ, Meza Santoscoy PL, Harrison MR, Fox J, Parkin CA, Ingham PW, Cunliffe VT: Identification of compounds with anti-convulsant properties in a zebrafish model of epileptic seizures. Dis Model Mech 2012, 5:773-784.

35. Yasmin L, Kinoshita S, Asaduzzaman M, Akolkar DB, Ikeda D, Ono Y, Watabe S: A 5'-flanking region of embryonic-type myosin heavy chain gene, MYH(M) (7)(4)(3)(-)(2), from torafugu Takifugu rubripes regulates developmental muscle-specific expression. Comp Biochem Physiol Part D Genomics Proteomics 2011, 6:76-81.

36. Parmacek MS, Ip HS, Jung F, Shen T, Martin JF, Vora AJ, Olson EN, Leiden JM: A novel myogenic regulatory circuit controls slow/cardiac troponin C gene transcription in skeletal muscle. Mol Cell Biol 1994, 14:1870-1885.

37. Meng X, Noyes MB, Zhu LJ, Lawson ND, Wolfe SA: Targeted gene inactivation in zebrafish using engineered zinc-finger nucleases. Nat Biotechnol 2008, 26:695-701.

38. Nakai J, Ohkura M, Imoto K: A high signal-to-noise $\mathrm{Ca}(2+)$ probe composed of a single green fluorescent protein. Nat Biotechnol 2001, 19:137-141.

39. Tian L, Hires SA, Mao T, Huber D, Chiappe ME, Chalasani SH, Petreanu L, Akerboom J, McKinney SA, Schreiter ER, Bargmann Cl, Jayaraman V, Svoboda K, Looger L: Imaging neural activity in worms, flies and mice with improved GCaMP calcium indicators. Nat Methods 2009, 6:875-881.

40. Amores A, Force A, Yan YL, Joly L, Amemiya C, Fritz A, Ho RK, Langeland J, Prince V, Wang YL, Westerfield M, Ekker M, Postlethwait JH: Zebrafish hox clusters and vertebrate genome evolution. Science 1998, 282:1711-1714.

41. Koopman P, Schepers G, Brenner S, Venkatesh B: Origin and diversity of the SOX transcription factor gene family: genome-wide analysis in Fugu rubripes. Gene 2004, 328:177-186.

42. Han Y, Lefebvre V: L-Sox 5 and Sox6 drive expression of the aggrecan gene in cartilage by securing binding of Sox 9 to a far-upstream enhancer. Mol Cell Biol 2008, 28:4999-5013.

43. Lefebvre V, Li P, de Crombrugghe B: A new long form of Sox5 (L-Sox5), Sox6 and Sox9 are coexpressed in chondrogenesis and cooperatively activate the type II collagen gene. EMBO J 1998, 17:5718-5733.

44. Smits P, Li P, Mandel J, Zhang Z, Deng JM, Behringer RR, de Crombrugghe $B$, Lefebvre $V$ : The transcription factors L-Sox 5 and Sox6 are essential for cartilage formation. Dev Cell 2001, 1:277-290.

45. Bessarab DA, Chong SW, Srinivas BP, Korzh V: Six1a is required for the onset of fast muscle differentiation in zebrafish. Dev Biol 2008, 323:216-228.

46. Maves L, Waskiewicz AJ, Paul B, Cao Y, Tyler A, Moens CB, Tapscott SJ: Pbx homeodomain proteins direct Myod activity to promote fast-muscle differentiation. Development 2007, 134:3371-3382.

47. Hirata H, Saint-Amant L, Waterbury J, Cui W, Zhou W, Li Q, Goldman D, Granato M, Kuwada JY: Accordion, a zebrafish behavioral mutant, has a muscle relaxation defect due to a mutation in the ATPase Ca2+ pump SERCA1. Development 2004, 131:5457-5468.

48. Smits $P$, Lefebvre $V$ : Sox 5 and Sox 6 are required for notochord extracellular matrix sheath formation, notochord cell survival and development of the nucleus pulposus of intervertebral discs. Development 2003, 130:1135-1148.

\section{Submit your next manuscript to BioMed Central and take full advantage of:}

- Convenient online submission

- Thorough peer review

- No space constraints or color figure charges

- Immediate publication on acceptance

- Inclusion in PubMed, CAS, Scopus and Google Scholar

- Research which is freely available for redistribution 